\title{
Hierarchical Settlement Networks
}

\begin{abstract}
A network representation is introduced for visualizing hierarchical region structures on various spatial scales. The method is based on a spring-block model approach borrowed from physics; it was previously used successfully to detect regions in any geographical space. Transylvania, USA and Hungary are used to demonstrate the network construction method.
\end{abstract}

Keywords: settlement networks, spring-block model, USA, Transylvania, Hungary.

\section{Introduction}

It is commonly believed that the core of any complex system is a network (Barabási 2005). The underlying network structure carries useful information for understanding the evolution and measurable characteristics of large systems. Networks have proven that they are useful for studying biological, social and economic systems (Barabási 2012).

A network approach might also be helpful in visualizing and understanding the complex hierarchical inter-relationship between settlements in a given geographic region. The method for such an approach is straightforward, assuming that hierarchical region-like structures and region centres on different spatial scales are detectable. The hierarchical space-division at various scales can be synthesized in a compact and visually interpretable manner by drawing up such a tree-like network topology. Here, based on a previously the elaborated region detection algorithm (Máté-Néda-Benedek 2011), a method for the construction of such a hierarchical connection network is presented.

This method can be helpful in the spatial analysis of all types of territorial data. The complexity of socio-economic processes with spatial dimension implies that natural space delimitation cannot be possible (Dusek 2004); the determination of territorial units (regions) depends on the examined phenomenon and its geographical characteristics (Haggett 2001). When researching the spatial flow of goods, services and information, it is possible to border regions with different spatial extent in different hierarchical structures. Thus, it is highly important in bordering to clarify the regional structures on micro, mezzo and macro regional levels, based on different socio-economic data.

a Institute for Theoretical Physics, Heidelberg University, Philosophenweg 16 D-69120 Heidelberg, Germany E-mail: G.Mate@tphys.Uni-Heidelberg.DE

b Edutus College, Department of International Business, 1114 Budapest, Villányi út 11-13., Hungary, E-mail: kovacs.andras@edutus.hu

c Department of Physics, Babes-Bolyai University, str. Kogalniceanu 1, R0-400084 Cluj-Napoca, , Romania, E-mail: zneda@phys.ubbcluj.ro 
The method enables us to border regions on different spatial levels by using different kinds of data. This flexibility may contribute to a more efficient regional research in bordering and in the horizontal and vertical division of socio-economic and geographical space (Nemes Nagy 2009).

This paper is divided into three main parts. First the methodological background and its geographical relevance is introduce; in the second part, we introduce three examples where spatial data are analysed and visualized (examples are from the USA, Transylvania and Hungary); in the last part, the socio-economic relevancies of this method in spatial analysis and regional science are explained.

\section{Method}

\section{Preliminaries}

Spring-block models have been widely used to model various phenomena related to relaxation, avalanche-like dynamics or self-organized criticality (Járai-Szabó-Néda 2012, Kovács-Néda 2007). The model considers a system of blocks that are interconnected by springs in a lattice like topology. The blocks can slide on a surface, and apart from the elastic forces experienced from their neighbours, a friction force is damping their free movement on the surface. The friction forces are usually velocity dependent. In the simplest version, there is a dynamic friction force that is independent of the sliding block's velocity and a static friction force (acting when the block is in rest relative to the surface), which has a larger value than the dynamic one. Originally, the model was considered in one-dimension by Burridge and Knopoff in order to model the power-law like distribution of earth-quakes magnitude (Burridge-Knopoff 1967). The model was then extended to two-dimensions by Olami, Feder and Christensen (1992). One of the most spectacular applications of this model is its success in modelling fracture and fragmentation patterns resulting from quasi-static drying of granular materials in contact with a frictional substrate (Leung-Néda 2010). The success of reproducing this relaxation dynamics in drying granular materials motivated the use of the spring-block model for hierarchical region detection. Regions can be imagined as resulting from a fragmentation dynamic in a geographic space. Once the interconnection topology of the settlements is defined, and the strength of the interconnecting forces is revealed, a simple relaxation dynamics will lead us to a hierarchical grouping. This hierarchical regiondetection method was used in "Máté-Néda-Benedek 2011". Here, for the sake of an easier understanding of the network construction method, the main elements in the spring-block approach for region detection are briefly reviewed. For a more detailed description, refer to "Máté-Néda-Benedek 2011".

\section{The spring-block model for region detection}

Settlements are regarded as blocks with masses $m_{i}$ proportional with their population $\left(W_{i}\right)$. The blocks are displaced on a two-dimensional plane so that their relative coordinates are determined by the original GPS coordinates of the settlements. On each block " $i$ " a friction force is acting on behalf of the surface. For the sake of simplicity, we 
consider here an over-damped motion of the blocks. The value of the force acting on block " $i$ " is defined in analogy with classical mechanics:

$$
F_{i}^{f}=m_{i} g \mu
$$

Here, $g$ is a hypothetical gravitational constant, taken as $g=1$ (defining the units for forces), and $\mu$ is the static friction coefficient.

The blocks are interconnected by springs. In order to determine the interconnection topology, we use a Delaunay triangulation (Okabe-Boots-Sugihara-Chiu 2000). The Delaunay triangulation performed on the point-like coordinates of the settlements will yield the first-order neighbours (Figure 1a). Springs are placed between these first-order neighbours, and as a result, a two-dimensional spring-block lattice will form (Figure 1b). Relaxation is performed on this spring-block lattice.

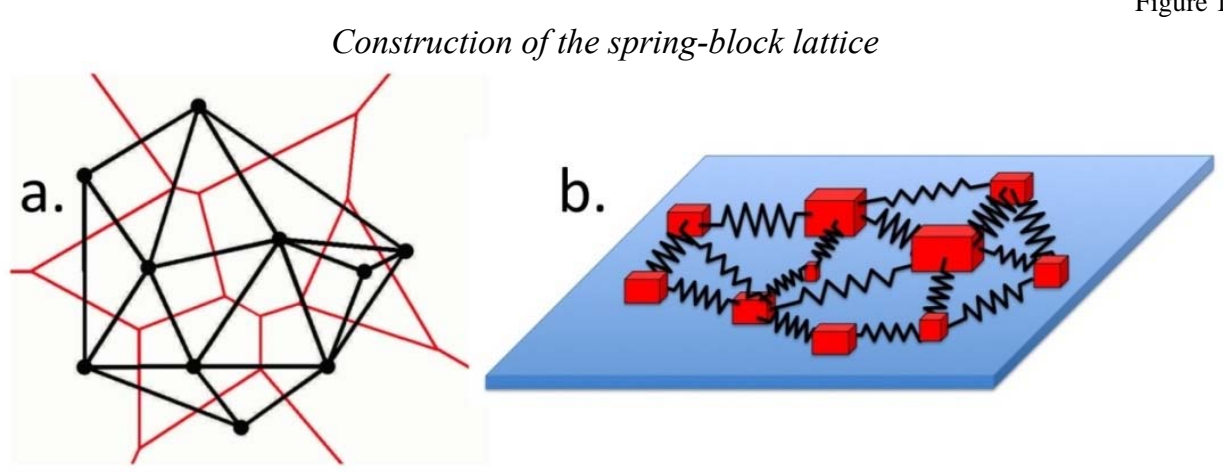

a) Delaunay triangulation for detecting the neighbours of the settlements. Black dots represent the position of the settlements; red polygons are the Voronoi cells around them and the black lines illustrate the Delaunay triangulation. We connect by springs the points connected by these edges.

b) A two-dimensional spring-block system. Red blocks represent settlements of different sizes and the springs connect the neighbouring blocks detected through the Delaunay triangulation.

Springs are modelling the socio-economic connections between the neighbouring settlements. Settlements that are closer to each other should attract each other in a stronger manner; settlements that have a correlated tendency of evolution for some of their relevant socio-economic indicators should also attract each other in a strong manner. Taking into account these obvious facts, the spring-force between settlements “i” and " $j$ " is quantified as:

$$
\overrightarrow{F_{i j}^{s}}=-\frac{k_{i j}}{d_{i j}} \overrightarrow{\varepsilon_{i j}}
$$

In the above equation $k_{i j}$ denotes the connectivity strength between settlements " $i$ " and " $j$ ", $d_{i j}$ is the distance between them and $\overrightarrow{\varepsilon_{i j}}$ is a unit vector pointing from one settlement to the other. In contrast with many earlier spring-blocks models, here the spring-forces are not linear. The tension in the spring is increasing while the distance between the settlements gets smaller. Another difference relative to the classical spring-block models is that here the springs cannot break, and they are able to resist any tension.

The crucial point in defining the spring-block model is to quantify the $k_{i j}$ connectivity strength. In order to achieve this, we consider a relevant socio-economic parameter, 
$q_{i}(t)$, which is available on settlement level for a relatively long time-period, t. This can be, for example, the population of the settlement, or income. Furthermore, we suppose that we do have access to this data with a uniform time-sampling interval, taken here as unity. We can define in such manner for each settlement the relative change of this quantity for a unit time:

$$
r_{i}(t)=\frac{q_{i}(t+1)-q_{i}(t)}{q_{i}(t)}
$$

The main hypothesis for the spring-block approach is that the time variation of the $r_{-} i$ relative changes has to be strongly correlated for the relevantly connected settlements. This means that we can quantify the $k_{i j}$ connection strengths as the time-like correlations between the $r_{i}$ quantities. Using Pearson correlations, we assume thus:

$$
k_{i j}=\left|\frac{\left\langle r_{i}(t) r_{j}(t)\right\rangle_{t}-\left\langle r_{i}(t)\right\rangle_{t}\left\langle r_{j}(t)\right\rangle_{t}}{\sigma\left[r_{i}(t)\right] \sigma\left[r_{j}(t)\right]}\right|
$$

Here, \langle\rangle , stands for an average taken over time, and $\sigma$ is the standard deviation:

$$
\sigma\left[r_{i}(t)\right]=\sqrt{\left\langle r_{i}^{2}(t)\right\rangle_{t}-\left\langle r_{i}(t)\right\rangle_{t}^{2}}
$$

Please note the absolute value on the right side of equation (4). This is introduced due to the fact that $k_{i j}$ must be positive, and both a high correlation and anti-correlation indicate a strong connection between settlements " $i$ " and “ $j$ ”.

\section{The Dynamics}

Once the main elements of the model and their relation to the relevant socio-economic and geographic data are clarified, we sketch the dynamics that lead to the hierarchical territory division.

1. Initially the settlements are placed on a plane according to their GPS coordinates. The $m_{i}$ mass of each block is fixed according to the population of the corresponding settlement $\left(m_{i}=W_{i}\right)$. Neighbours are defined with the Delaunay triangulation and springs are inserted between them. The $d_{i j}$ distances between the neighbouring settlements and their $k_{i j}$ connectivity strength is calculated and fixed for the whole duration of the simulation. The resultant elastic force acting on each block is computed: $\overrightarrow{F_{l}^{s}}=\sum_{j(i)} \overrightarrow{F_{l j}^{s}}$

2. In the equation above, we denoted by $j(i)$ the neighbours of block $i$.

3. The friction coefficient is fixed to a large value so that no resultant force acting on the blocks exceeds the friction force $\forall i \rightarrow\left|\overrightarrow{F_{l}^{s}}\right|<F_{i}^{f}$ :

4. We decrease the value of $\mu$, until the first block is allowed to slide, i.e. the magnitude of the resultant tension force is bigger than the static friction force acting on the block $\left(\left|\overrightarrow{F_{l}^{s}}\right|>F_{i}^{f}\right)$.

5. The block "i" in one simulation step will be moved by a distance $h$ in the direction of the $\overrightarrow{F_{l}^{s}}$ force. The $h$ value is chosen to be much smaller than the smallest initial distance between any two settlements. As a result of this slip, if any two blocks come closer to each other than a predefined $d_{\min }$ value, these blocks are united in one cluster. This cluster will be a new block in the system, inheriting the mass and links of both blocks, and in the next simulation steps will 
represent all the blocks that are glued together in it as a result of this coalescence process.

6. After each move, the distances among the moving blocks and its neighbours are recalculated.

7. In the next simulation step, the moving block and its neighbouring blocks are checked whether they satisfy the slipping condition. If yes, they are moved by a distance $\mathrm{h}$ in the direction of the resultant forces. This simulation step is repeated until no block can move. i.e. $\forall \mathrm{i} \rightarrow \overrightarrow{\left|F_{l}^{s}\right|}<\mathrm{F}_{\mathrm{i}}^{\mathrm{f}}$

8. The movement of blocks will continue in the consecutive simulation steps until the condition $\overrightarrow{\left|F_{l}^{s}\right|}>F_{i}^{f}$ is satisfied for the new position.

9. When no more blocks can move, the $\mu$ friction coefficient is lowered until the first block satisfies the slipping condition again. The simulation proceeds from here by repeating the steps from 5 .

10. The dynamics ends when all blocks have collapsed into one.

The model and dynamics defined above has only two freely adjustable parameters. One is the $h$ distance of the slip in one simulation step, and the other is the $d_{\min }$ distance for the collapse of two blocks. If we use small enough values, (as described earlier), their value will not influence the hierarchical collapsing dynamics and the method will become free of adjustable parameters.

\section{Hierarchical territory division}

The above algorithm will hierarchically group the elements, respecting their connection strength with neighbours and their initial spatiality. Smaller blocks will slide towards large ones since the friction forces acting on the latter ones are larger. These large settlements will than become centres attracting other blocks. It is reasonable to assume that the previously presented spring-block relaxation dynamics will reveal regions at different scales as the system hierarchically collapses into larger and larger clusters. Keeping track of all these collapsing events, and by projecting backward in time the collapsing order, one can define a hierarchical clustering of the settlements for the investigated geographical region.

The method and model presented above was implemented in an interactive JAVA application (Máté-Néda-Benedek 2009), which allows the user to visually follow the whole clustering process up to the end when all blocks concur in one. The program memorizes all the intermediary situations and can interactively visualize the settlement partitions corresponding to them. One can freely play with this model by visiting the website dedicated to it (Máté-Néda-Benedek 2009).

\section{The hierarchical settlement connection network}

The collapsing dynamics obtained in the spring-block model can be used to draw a hierarchical network structure for the settlements. In order to construct this network, the hypothesis is that each cluster is dominated by the largest settlement inside it. This means that when two clusters collapse we assign a link between the dominating settlements. In order to visualize different levels of hierarchy and to obtain a visually interpretable 
network structure, we draw lines with different colours or grey-shades according to the collapsing stage. One possibility is to use the whole colour palette from blue to red according to the simulation time (step) of the time moment for the collapse. Blue lines will represent the initial stage of the collapse, and dark red lines the latest stages when only few clusters are present in the dynamics. In such manner, interpreting the resulted weighted graphs has to be carried out by taking into account the colour code illustrated on the attached legend. Another possibility is to use only warm colours, and indicate the collapse stage with a colour shifting from red to yellow. This convention generates more user-friendly networks, but it offers less resolution for illustrating different stages of the collapse. It is also possible to use grey-shades instead of colours. In such cases, the darker levels will correspond to earlier collapse time and lighter lines will indicate connections made on a bigger cluster level. We will illustrate all these methods in the examples.

\section{Data}

In order to obtain the connectivity strength $\left(k_{i j}\right)$ between the settlements, we need a uniformly interpreted long-term dataset for some relevant socio-economic parameter $\left(q_{i}(t)\right)$. The most convenient data in such sense is the population $W_{i}$ of the settlements. One might assume that the variations in the population data will reflect both cultural and economic aspects of the settlements, so it seems appropriate for a well-balanced and relevant socio-economic indicator. It also has the advantage that, in general, long-term census data is easily accessible for all geographic territories. We have gathered freely accessible data on the Internet for Transylvania on settlement level (Varga 2007) (11 census data between 1850-2002) and for USA on county level (University of Virginia Library n.y) (last 5 census). Due to the courtesy of Professor József Nemes Nagy, we obtained excellent data on settlement level for Hungary (11 census data from 1870 to 1970). In the case of Hungary, Professor Nemes Nagy also provided the average local income tax paid per inhabitant for 20 consecutive years (data between 1990-2009). This data also provided the possibility for using different socio-economic indicators for determining the $k_{i j}$ connectivity strength.

In order to initially position the blocks (settlements) on the simulation plane and to determine their neighbours, we also needed the GPS coordinates of the settlements. These coordinates are usually available in an electronic format. For Transylvania they came from (Astroforum n.y), and in the case of USA the data for the county centre is available at (Comcast n.y.). For Hungary, Professor Nemes Nagy provided the data.

Once all these data are electronically available, the spring-block model can be initialised and its hierarchical collapse will reveal the relevant network structure.

\section{Results}

An interactive version of the program running on Windows, Linux and MacOsX operation systems can be downloaded from (Máté-Néda-Benedek 2009). Once the Java Runtime Environment (JVE) is installed and correctly running, one can test the program. In the online version, it will be possible to select the studied territory (Transylvania, USA 
or Hungary), the type of data used for the connectivity (population, tax) and the visualization method. It is possible to visualize the "regions" obtained on different hierarchical levels by rewinding the collapsing scenario. An alternative to this is to plot the hierarchical network structure that is intended to synthesize visually the region structures obtained at various collapsing stages. Results obtained for Transylvania, USA and Hungary are given in Figures 2, 3 and 4.

For Transylvania we draw the edges using a colour code extended from blue to dark red (Figure 2). Someone who is familiar with the map of Transylvania will immediately spot on this graph, as local hubs (gathering the tree-like connection topology in their neighbourhoods), the main region capitals (Cluj-Napoca, Timisoara, Sibiu, Brasov, Oradea, Targu Mures and Baia Mare). In order to illustrate the topology of the network structure on a lower level, we have magnified the Banat region, with the obvious centre in Timisoara. This blow up indicates the hierarchical space division and the lower level geographical regions in Banat.

For USA, we have used a grey-scale code to illustrate the hierarchical network structure on a county level (Figure 3). Two regions (one in the neighbourhood of Memphis, Tennessee, and one in the neighbourhood of Salt Lake City, Utah) are magnified to show the fine structure.

For Hungary (Figure 4), we use yet another representation, by considering only warm colours ranging from red to yellow. Here we compare the settlement network structures obtained using two very different datasets. One network is based on long-term population census (from 1870 to 1970), and the other one is obtained by using data for 20 consecutive years (from 1990 to 2009) of income tax per inhabitant. Although the topology is statistically very similar, the two magnified regions show that the obtained network structures are quite different. This illustrates well that the hierarchical networks structures constructed in such manner are rather sensitive to the used data and the spanned time-period.

After the visualization of the results, the socio-economic implications will be taken into account. The most important advantage of the applied method lies in the followings:

- The relation between time processes and spatial distribution can be seen in a single map (see methodology). The spatial analysis and visualization of timebased data (time series) is possible with this method. It is based on long-term socio-economic data, and the method helps to discover the space-time relations by different variables. It can serve, for example, as the basis for the bordering of regions and gravity zones of city regions as the strengths and directions of linkages can be seen in a single map. These maps show the effect of geographical distance on regional structures.

- The level of spatial structure can be determined by the researcher during the visualization process. During the modelling procedure, the researcher has the opportunity to determine the desired number of formulating regions. It means that one can draft a multilevel regional structure by stopping the simulation at different stages. In the Transylvanian example, a solid vertical structure of micro, mezzo and macro regions can be observed (See the different colours and patterns.). 
The settlement connection network for Transylvania (Romania) obtained by using long-term population census data.

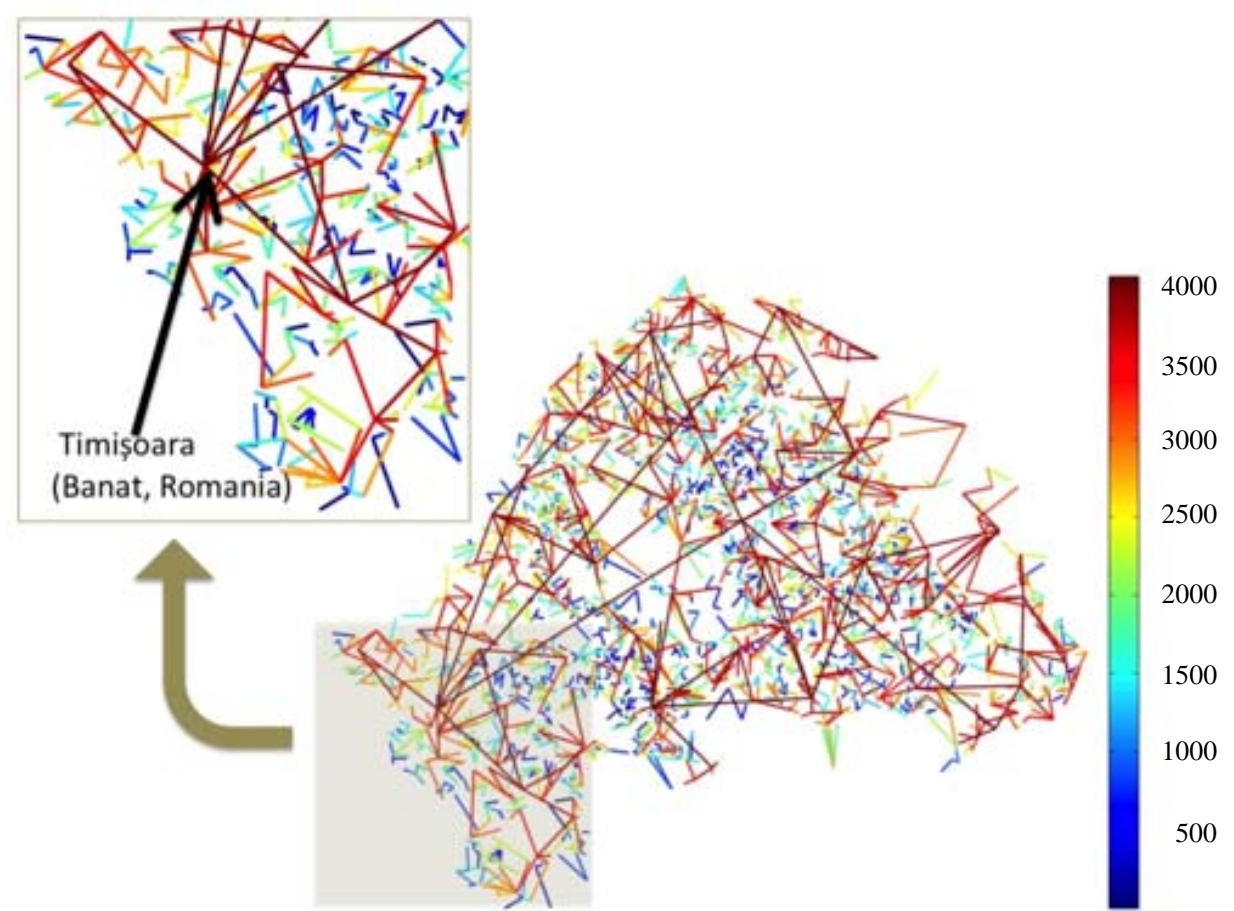

The colour of the lines indicates the simulation time when the collapse in the relaxation dynamics occurred. Colder colours are for earlier stages of the dynamics and warmer colours are for the later stages, as is indicated in the attached legend. The Banat region, with the obvious centre in Timisoara, is magnified to give a better visualization of the local nature of the obtained settlement network.

- The method is suitable for comparing many different kinds of data with socioeconomic relevance. For horizontal and vertical division of socio-economic space, strong spatial links and long-term processes are needed. This method allows us to compare the spatial linkages and strength in case of different variables. In the Hungarian example (where we had two variables to work with), we have the opportunity to compare the similarities and differences in spatial links by using population and income data. As one can see in Fig 4, there are considerable differences in the two variables. The maps on population and income have a partly different topology. The map on the census data shows stronger ties on a microregional scale than the map on per capita income. This may come from the different time dimensions of data, from the diverse behaviour of social and economic processes, etc. 
- The original settlement (or regional) structure of the researched area is depicted in the visualized network. The applied method uses GPS-data in the visualization process, so not only the strength of the link between the neighbouring settlements can be identified, but the real settlement network as well. In the maps of the USA and Hungary, differences in density and spatial distribution of settlement are easy to identify. In the case of Hungary, the small village areas of Somogy, Baranya and Cserehát can be distinguished from the Alföld region.

Figure 3

The county connection network revealed for USA by using population census data

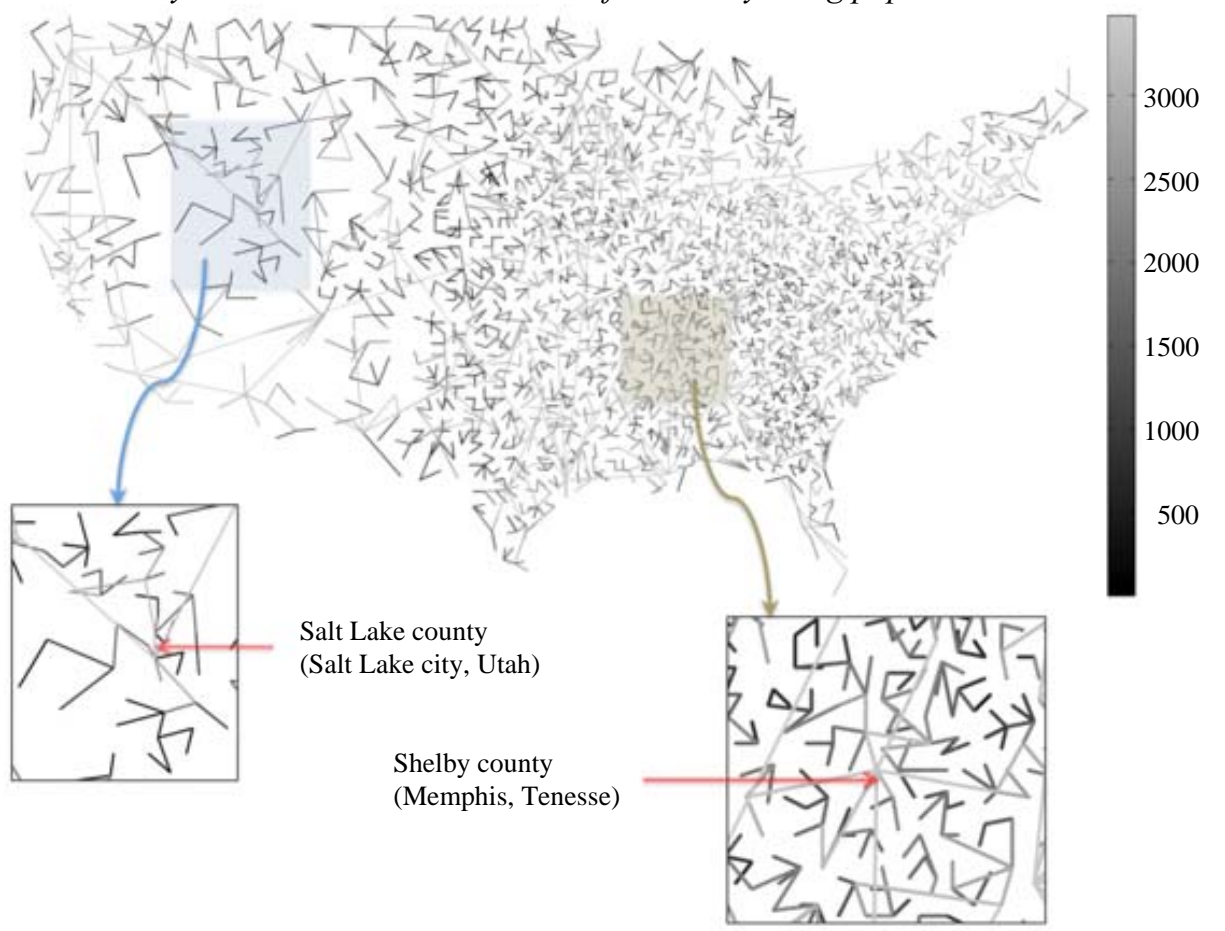

A grey-scale code (see the attached legend) indicates the simulation step when the collapse occurred in the relaxation dynamics. Two rectangular regions are magnified, exemplifying the local connectivity topology. 
The settlement connection network for Hungary constructed by using long-term population census data (top) and average income tax data for 20 consecutive years (bottom)

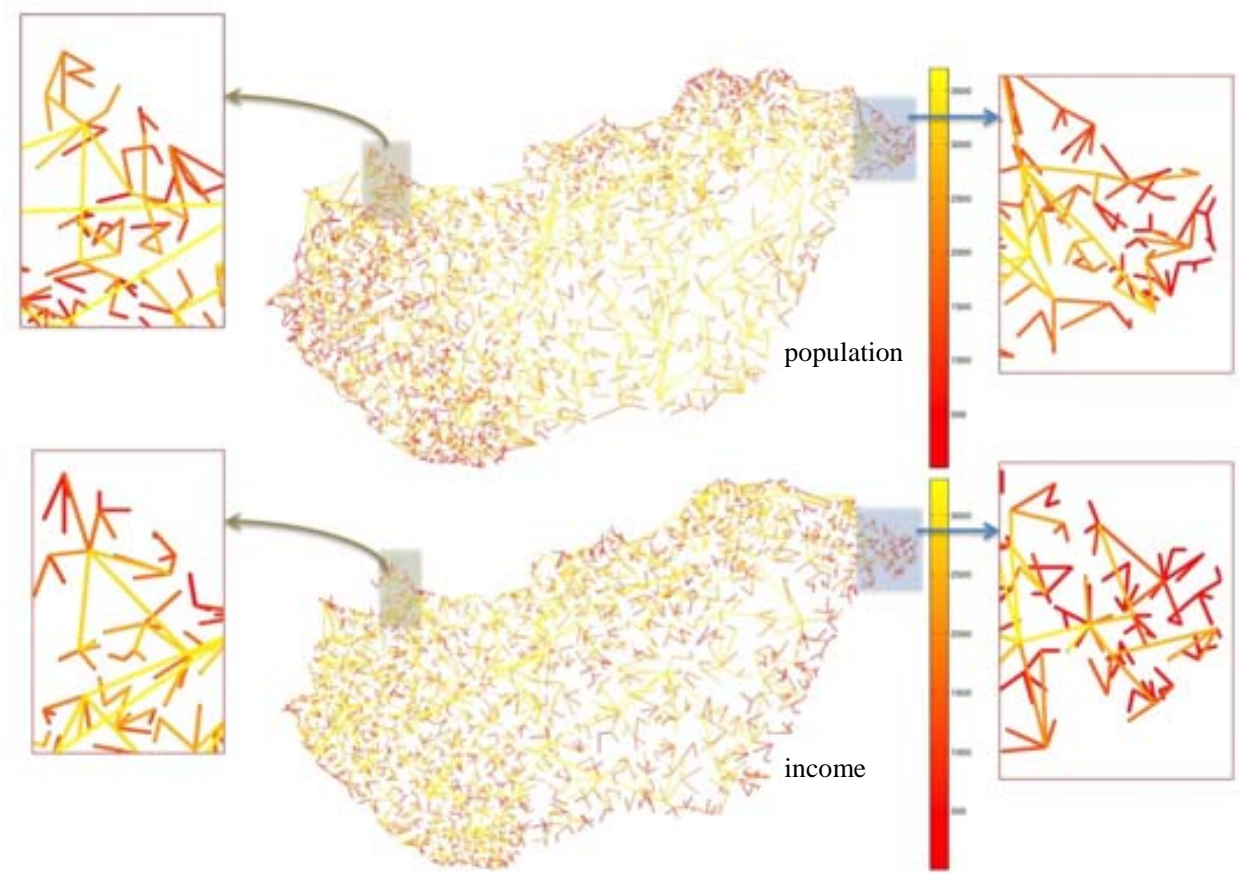

The color code indicates the simulation step when the collapse occurred in the relaxation dynamics. Lighter colors (in the direction of yellow) indicate later stages, as it is illustrated on the attached legends. Two regions are magnified. Although the topology looks statistically similar, the networks obtained with the different input data are very different.

\section{Conclusions}

The hierarchical settlement connection networks plotted in the present study offers an intuitive and visually appealing presentation for the complex inter-relationship between the settlements. Due to the used "link" definition, the network has a tree-like structure, and no loops are present. Settlements are connected only through the region centres revealed at different spatial scales. These centres act as hubs of different sizes. The shortest distance (minimal number of links) between any two nodes (settlements) will indicate how strongly these settlements are connected. The hierarchy levels are illustrated by using different colour codes. Results obtained for the examples of Transylvania, USA and Hungary were used to exemplify the method.

Our approach and the applied techniques may help the regional research in several fields. In regional and spatial planning it can contribute to the planning, realization and control phases for determining an optimal regional structure (in advance), to identify regions with special characteristics (backward areas, development axes, city regions, etc.) or to measure and visualize the changes in settlement networks. In business, it can 
contribute to the spatial analysis of market data like spatial relations in revenues, profit, costs, etc., to identify spatial distribution and concentration of business partners (customers, suppliers) or business network elements like market centres, industrial parks, clusters; or to find hierarchies in business activities (competitors, partners).

We believe that such representations could help in designing socio-economic structures that would optimise and better serve territorial organization and business activity.

\section{REFERENCES}

Barabási, A. L. (2005): Taming Complexity Nature Physics 1:68-70.

Barabási, A. L. (2012): The network take over Nature Physics 8: 14-16.

Máté, G. - Néda, Z. - Benedek, J. (2011): Spring-block models reveals region-like structures PLOS One 6: 16518.

Dusek; T (2004): A területi elemzések alapjai [Introduction to spatial analysis] Eötvös Lóránd University, Budapest.

Haggett; P. (2001): Geography: A global synthesis Pearson Education, Harlow.

Nemes Nagy, J. (2009): Terek, helyek, régiók [Spaces, Places, Regions] Akadémiai Kiadó, Budapest.

Járai-Szabó, F. - Néda, Z. (2012): Earthquake model describes traffic jams caused by imperfect driving styles Physica A 391 (22): 5727-5738.

Kovács, K. - Néda, Z. (2007): Disorder-driven phase transition in a spring-block type magnetization model Physics Letters A 361 (1-2): 18-23.

Burridge, R. - Knopoff; L. (1967): Model and theoretical seismicity Bulletin of Seismological Society of America 57 (3): 341-371.

Olami, Z. - Feder, J.S. - Christensen, K. (1992): Self-organized criticality in a continuous, nonconservative cellular automaton modelling earthquakes Physical Review Letters 68 (8): 1244-1247.

Leung, K. T. - Néda, Z. (2010): Criticality and pattern formation in fracture by residual stresses Physical Review E 82 (4): 046118

Okabe, A. - Boots, B. - Sugihara, K. - Chiu, S.N. (2000): Spatial Tessellations: Concepts and Applications of Voronoi Diagrams Wiley, Chichester.

Máté, G. - Néda, Z. - Benedek; J. (2009): Region detection homepage http://atom.ubbcluj.ro/ gmate/regions. Accessed 2012 Jun 3.

Varga, E. A. (2007): Erdély etnikai és felekezeti statisztikája [Ethnical and denominational statistics for Transylvania, Census data for 1850-2002] http://www.kia.hu/konyvtar/erdely/erd2002.htm. Accessed 2012 Jun 3.

University of Virginia Library (n.y): Historical census browser. http://fisher.lib. virginia.edu/collections/stats/histcensus/. Accessed 2011 Jan 6.

Astroforum (n.y): Coordonate de localităti din România [Coordinates of Romanian settlements] http:// www.astroforum.ro/index.php?showtopic=90. Accessed 2011 Jan 6.

Comcast (n.y.): Coordinates of counties in USA http://home.comcast.net/ rickking04/ gis/COUNTYSPC.XLS. Accessed 2012 Jun 3.

\section{Acknowledgment}

This research was supported in the framework of TÁMOP 4.2.4. A/2-11-1-2012-0001 'National Excellence Program - Elaborating and operating an inland student and researcher personal support system' project. The project was subsidized by the European Union and the State of Hungary. We thank Professor Jozsef Nemes Nagy for the provided data. 